A.J. Morales ${ }^{1}$, I.M. Lasky ${ }^{1}$, J. Reyes ${ }^{1}$, K.A. Ahmed ${ }^{1}$, I. Boxx ${ }^{2}$.

"Controlling Flame Stability in a High-Speed Combustor through Pressure

Gradients”, AIAA Scitech 2019 Forum, (AIAA 2019-1733).

${ }^{1}$ University of Central Florida, Orlando, Florida.

${ }^{2}$ Institute of Combustion Technology, German Aerospace Center. Stuttgart.

The AIAA version of the paper is accessible at https://doi.org/10.2514/6.2019-1733

On the AIAA web page

https://arc.aiaa.org/doi/pdf/10.2514/6.2019-1733

the interested reader can find other material published by AIAA 


\title{
Controlling Flame Stability in a High-Speed Combustor through Pressure Gradients
}

\author{
Anthony J. Morales*, Ian M. Lasky ${ }^{\dagger}$, Jonathan Reyes ${ }^{\ddagger}$, and Kareem A. Ahmed ${ }^{\S}$ \\ University of Central Florida, Orlando, FL, 32816 \\ Isaac Boxx ${ }^{\text {II }}$ \\ Deutsches Zentrum für Luft-und Raumfahrt, Cologne, Germany
}

\begin{abstract}
Flame-vortex dynamics are investigated within a premixed blow-down combustion facility during lean blowout. Bluff body flames are stabilized within a variable geometry test section that allows for the downstream pressure gradients to be altered via manipulation of the external walls. Lean blowout is then induced by removing the fuel flow into the reactant mixture. The blowout process is captured using simultaneous high-speed particle imaging velocimetry (PIV) and $\mathrm{C}_{2} * \mathrm{CH}^{*}$ species measurements. This combination allows for temporal analysis of both the flow field and the reacting equivalence ratio throughout extinction. The temporal evolution of the flame structure, reacting flow vorticity, and the resulting strain rate along the flame are analyzed for three downstream geometry configurations to fully discern and control the hydrodynamic mechanisms of flame blowout. It is determined that the reduction of flame-generated baroclinic torque and an increase in downstream shear layer vorticity are the primary sources of flame blowout. Modifying the downstream pressure gradient will change baroclinic torque production, allowing for attenuation or augmentation of the vorticity mechanisms associated with lean blowout.
\end{abstract}

\section{Nomenclature}

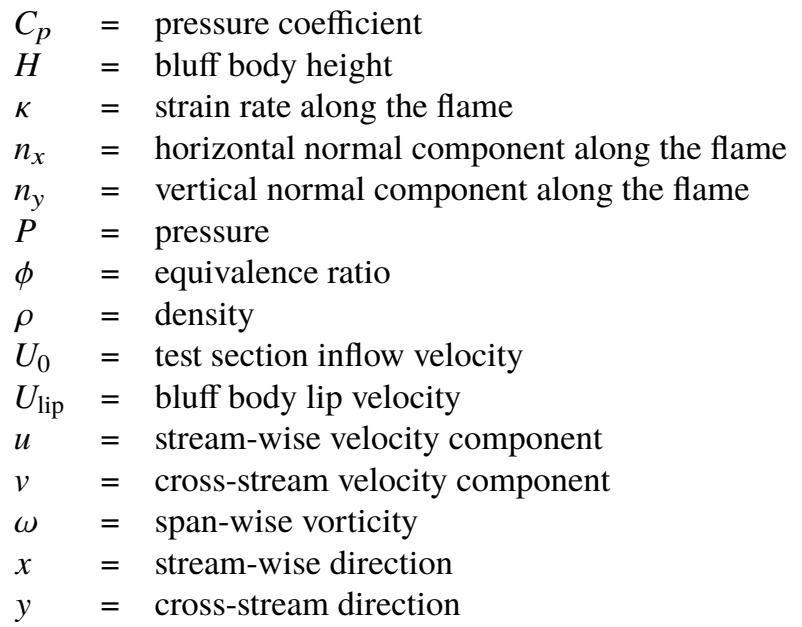

\footnotetext{
*Graduate Research Assistant, University of Central Florida, Bldg. 154, Rm. 109, AIAA student member.

†Undergraduate Researcher, University of Central Florida, Bldg. 154, Rm. 109, AIAA student member.

$\doteqdot$ Graduate Research Assistant, University of Central Florida, Bldg. 154, Rm. 109, AIAA student member.

$\S$ Assistant Professor, Mechanical and Aerospace Engineering,University of Central Florida, ENGR 1, Rm. 410, AIAA member.

I Ph. D, Senior scientist at the Institute of Combustion Technology of the German Aerospace Center.
} 


\section{Introduction}

$\mathrm{C}^{\mathrm{LAME}}$ extinction is a critical issue that continues to hinder the performance of combustion-driven propulsion engines

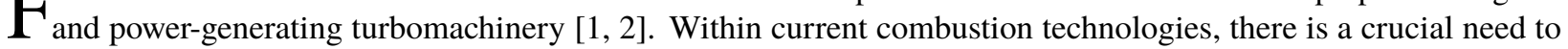
improve energy conversion efficiencies while optimizing fuel efficiency, reducing harmful emissions, and maintaining flame stability [3]. To accomplish this, lean combustion can be implemented to save fuel costs and reduce $\mathrm{NO}_{\mathrm{x}}$ emissions. However, operating at lean equivalence ratios induces hydrodynamic instabilities within the reacting flow field that compromises flame stability and can lead to flame blowout [4, 5]. Therefore, continued study of flame-vortex interactions will lead to improved understanding of the transient instabilities causing lean blowout and ultimately aid in the advancement of future combustion technologies.

Bluff bodies are commonly placed within a bulk flow to provide the necessary fluid dynamics to achieve flame ignition [1, 6, 7]. The immediate bluff body wake consist of a recirculation zone bounded by shear layers of high-magnitude vorticity. The shear layers and recirculation zone promote stable combustion through continuous turbulent mixing between the cold inflow reactants and hot combustion products [7-10]. Once ignition occurs, combustion driven vortex mechanisms have profound effects of the reacting flow field. For instance, combustion heat release induces gaseous expansion, providing thrust augmentation from flow dilatation [11]. Additionally, the reaction induces baroclinic torque production along the flame boundary [12]. The baroclinic torque manifests from the misalignment of the downstream pressure gradient and the density gradient between the free-stream reactants and combustion products [11, 13, 14]. In the far downstream bluff body wake region, the vorticity field and flame structure are dominated by the baroclinic torque mechanism. Therefore, manipulation of this mechanism will affect overall flame structure and stability.

When operating at lean equivalence ratios, the combustion driven vorticity mechanisms are weakened and the flame becomes susceptible to hydrodynamic instabilities. Near the lean blowout limit, researchers have hypothesized that reduced baroclinic torque magnitudes allow for a growth in downstream shear layer vorticity [14, 15]. The increased shear layer vorticity is also coupled with a decreased flame speed [16], allowing for a more pronounced interaction between the flame boundary and the bluff body shear layer [16-18]. Increased interaction with the shear layer promotes high strain along the flame boundary, stretching the flame beyond its allowable tolerance, and causing localized extinctions [13, 19]. Local flame extinctions result in increased reactant entrainment into the recirculation zone, reducing the temperature within the immediate wake, thereby weakening the combustion stabilization process.

As the reacting vorticity mechanisms are weakened, global bluff body instabilities tend to manifest. Emerson et al. has investigated this by varying the density ratio between the inflow reactants and combustion products and demonstrating that reduced baroclinicity leads to asymmetric flame wrinkling [15]. Asymmetry is also prevalent within lean reacting conditions where flames experience a sinuous structure reminiscent of Bénard-von Kármán (BVK) vortex shedding [19]. The BVK shedding is an absolute instability associated with nonreacting bluff body flows where opposing vortices within the recirculation zone will undergo periodic shedding and convect through the downstream wake [14, 20, 21]. The transition from stable burning to a global asymmetric oscillation is specifically attributed to reduced dilatation and baroclinicity [22]. This results in high curvature and velocity fluctuations along the flame boundary as it oscillates within the flow field [19]. This further stretches the flame and can result in localized extinctions that lead to global blowout. Based on these studies, it is highly evident that vorticity dynamics are responsible for increased instabilities when operating at lean conditions.

To further understand the influence of vorticity dynamics on flame stability, the current work seeks to explore the effect of changing the magnitudes of the combustion-induced vorticity mechanisms on an extinguishing flame-flow field. This is accomplished within a blow-down turbulent combustion facility utilizing a bluff body flame stabilizer. The vorticity mechanisms are altered by changing the downstream pressure gradient within the combustion test section via manipulation of the external wall geometry. Three downstream wall configurations are used to alter the pressure gradient: a straight, converging, and diverging configuration (referred to a nominal, nozzle, and diffuser configuration, within this work). Pressure gradient tailoring will allow for the manipulation of the flow field vorticity dynamics by altering the flame generated baroclinic torque. [15, 23, 24]. High-speed PIV diagnostics and $\mathrm{C}_{2}{ }^{*}-\mathrm{CH}^{*}$ species measurements allow for the baroclinic torque, as well as other vorticity mechanisms, to be spatially and temporally resolved throughout flame extinction. This analysis will allow for further understanding of flame extinction and provide additional insights regarding the controllability of vortex induced extinction mechanisms. 


\section{Experimental Method}

\section{A. Experimental Facility}

Flame blowout is investigated within a blow-down combustion facility. The combustion facility consists of a flow-conditioning plenum and combustion test section as shown in Fig. 1. High pressure air is ducted into the plenum and regulated with a Preso Venturi flow meter, Dwyer pressure transducer, and DM4500 series pneumatic JFlow valve. The bulk flow rate through the plenum is regulated with a feedback loop in conjunction with LabVIEW controlled software. Gaseous propane is injected upstream of the plenum to ensure a uniform mixture reaches the test section. The propane flow rate is controlled with an Omega FL-2092 pressure regulator and automated shut-off valve allowing for the desired equivalence ratios to be achieved. Four wire mesh screens are also installed along the length of the plenum to remove bulk flow instabilities.

The test section is comprised of a turbulence generator, a bluff body flame stabilizer, and an optical viewing section. The test section, bluff body, and downstream viewing region are shown in Fig. 2. The turbulence generator combines static grid and active fluidic jet impingement methods for a wide range of turbulence modulation. Detailed characteristics of the turbulence generator are documented in previous works [23-26]. The bluff body is ballistic in design and is vertically centered within the test section. The bluff body length and height are $64 \mathrm{~mm}$ and $16 \mathrm{~mm}$, respectively, and the width spans the entire width of the test section.

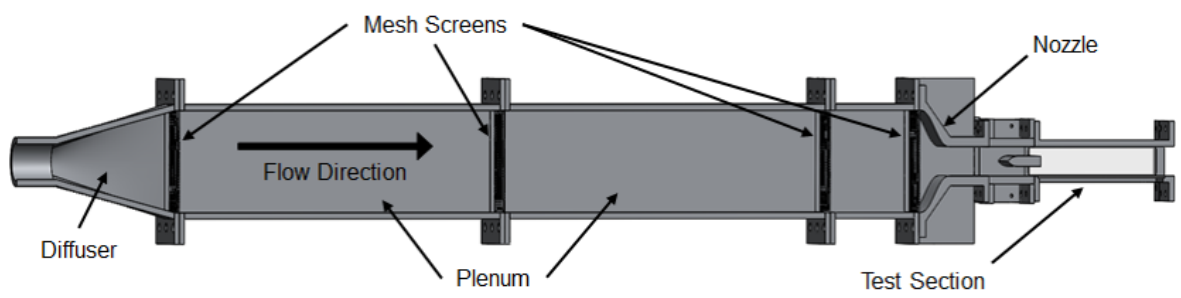

(a)

Fig. 1 Flow conditioning plenum and test section.

The optical viewing section is rectangular with an aspect ratio of 2.8:1. The bottom and side walls are fitted with fused quartz glass windows allowing for optical access into the test section. The top wall is not optically accessible and is painted black to minimize light scattering within the optical section. This wall is also fitted with static pressure taps, allowing for downstream pressure measurements to be made. The downstream pressure is modulated by manipulation of the top and bottom walls of the test section. Three degree angled wedges allow for three configurations to be achieved: nominal, nozzle, and diffuser. Each configuration is characterized by downstream pressure measurements, allowing for a downstream pressure gradient to be determined.

\section{B. Experimental Diagnostics and Extinction Analysis}

Simultaneous PIV and $\mathrm{C}_{2}^{*}$ - $\mathrm{CH}^{*}$ species measurements are used to analyze the extinction event. A dual-head ND:YAG $532 \mathrm{~nm}$ laser is used for PIV diagnostics. A pressurized swirl seeder is used to inject $150 \mathrm{~nm}$ aluminum oxide particles upstream of the flow plenum and used for the PIV measurement. The laser is synchronized with a Photron Fastcam SA.1.1 high-speed camera by means of a function generator. The entire PIV system operates

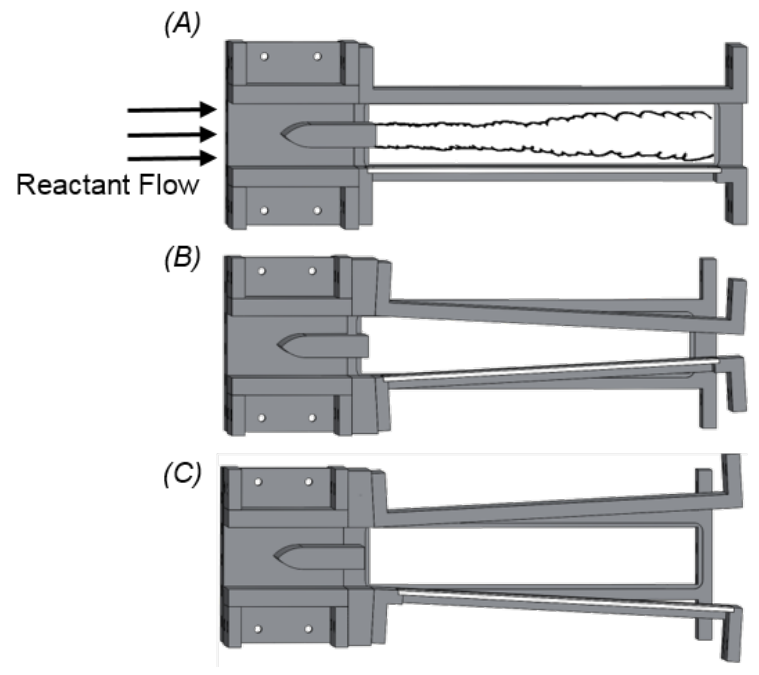

Fig. 2 Bluff body and optically accessible test section configurations. (A) Nominal, (B) Nozzle, and (C) Diffuser configurations. 
at $20 \mathrm{kHz}$, allowing for a temporal analysis of the flame

structure and flow field evolution.

The $\mathrm{C}_{2}^{*}-\mathrm{CH}^{*}$ species measurements are synchronized with the PIV measurement. A Photron SA1.1 fitted with a 200 mm F3.5 Nikon lens is used to capture the reacting flow species. The chemiluminescence of $\mathrm{C}_{2}, \mathrm{CH}$, and $\mathrm{OH}$ radicals have been previously used to determine species concentrations within reacting flows [27]. As the equivalence ratio changes, the amount of radiation emitted from the species will change; allowing for a correlation between $\mathrm{C}_{2}^{*}-\mathrm{CH}^{*}$ intensity ratios and the reacting equivalence ratio to be determined [27,-29].

In addition to the species measurements, the downstream pressure gradient is determined from reacting pressure measurements. The static pressure taps along the top wall of the optical viewing region allow for the use of a Scanivalve (PDCR23D) pressure transducer system to acquire downstream static pressure measurements. 3000 pressure measurements are taken at each location at frequency of $2500 \mathrm{~Hz}$. Data at each point is averaged to get a single average pressure measurement. The pressure measurements are accurate to $\pm 7 \mathrm{~Pa}$ per specifications. These measurements allow for the downstream pressure gradient to be quantified. When combined with the species measurements, the span-wise baroclinic torque can be temporally evaluated along the flame throughout blowout.

Inflow reactants initially enter the test section at an equivalence ratio of $\phi=0.7$. Once stable burning is achieved, extinction is induced by stopping the flow of fuel (gaseous propane) into the reactant mixture. The $\mathrm{PIV}$ and $\mathrm{C}_{2} * \mathrm{CH}^{*}$ species are used to characterize the lean blowout event. The boundary of the flame is determined from the PIV measurement and local velocity components, vorticity, and strain rates are extracted along this flame boundary. The experimental diagnostics allow for temporal analysis of the vorticity dynamics within the flow field while investigating the effects of pressure gradient tailoring on the mechanism of lean flame blowout.

\section{Results}

Flame extinction is investigated for the three wall configurations mentioned. For each configuration, the freestream velocity was maintained at $25 \mathrm{~m} / \mathrm{s}$, corresponding to a Reynolds number $(R e=\rho u H / v)$ of 25,200 , and the equivalence ratio decreases from $\phi=0.7$ to $\phi=0$. Temporal extinction results are presented using a normalized time variable, $t^{*}$, where $t^{*}=0$ represents the beginning of the extinction event and $t^{*}=1$ is where the entire flame blows out.

The downstream pressure measurements for all three wall configurations are shown in Fig. 3. The nozzle configuration produces the largest magnitudes of pressure within the immediate bluff body wake region. Additionally, the axial pressure decreases most rapidly for the nozzle configuration due to the reduction of cross-sectional area downstream resulting in the most significant acceleration of the flow. In contrast, the diffuser configuration experiences the smallest pressure magnitudes within the immediate bluff body wake and a minuscule decrease in downstream pressure because of the acceleration of the combustion products through an increasing axial cross-sectional area. For all three configurations, the global pressure gradient is $(\overline{d P / d x})$ defined as the total range of pressure divided by the total axial distance. Since the nozzle configuration experiences the largest decrease in downstream pressure, it produces the largest favorable global pressure gradient within the test section while the diffuser produces the smallest global pressure gradient (the nominal configuration resides between the two).

Lean flame extinction is analyzed using the three downstream test section configurations to understand the effect of the differing pressure gradients on the extinction process. The flow field, flame structure, and local strain rates along the flame are analyzed for each pressure configuration. An example of this is depicted within Figure 4 , which

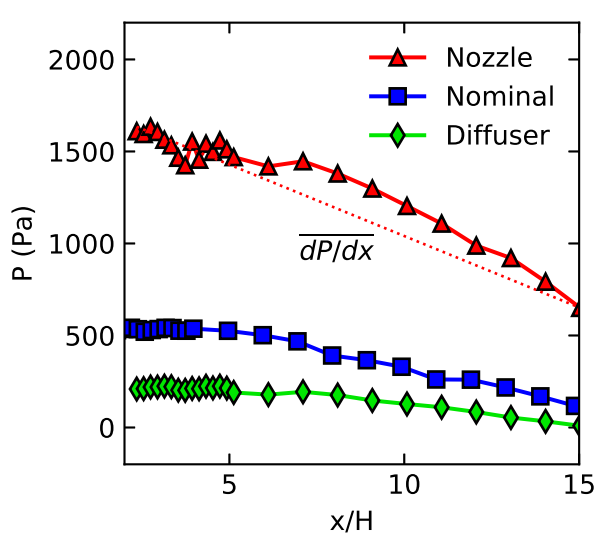
shows change in the flame-flow field for the nominal configuration as the flame extinguishes. As the flow field approaches complete blowout, the flame structure becomes asymmetrical and the flame span decreases. Furthermore, the vorticity within the flow field, which is produced from the bluff body shear layers also grows in magnitude as blowout is approached. The decreasing flame span and increased vorticity magnitudes are coupled with larger strain rate magnitudes along the 

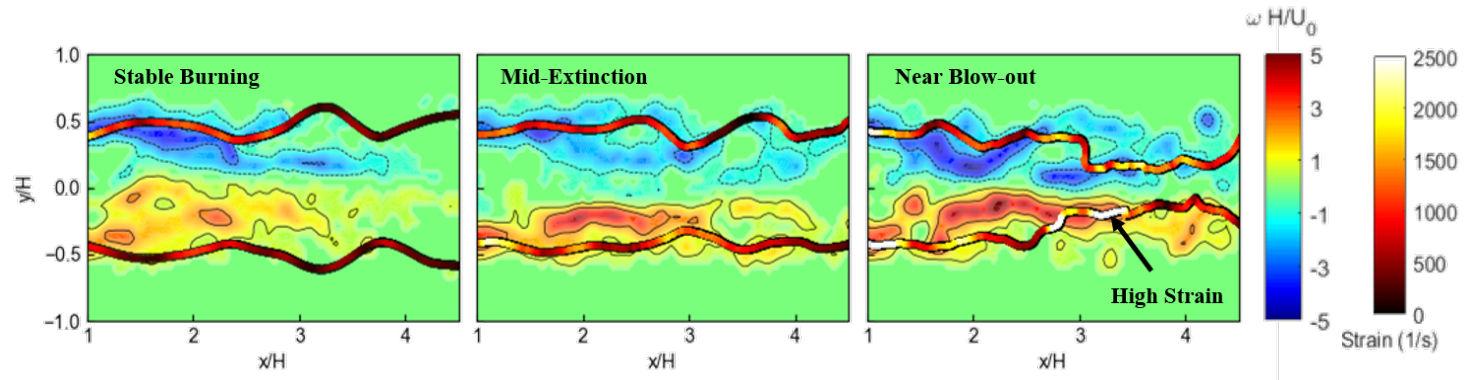

Fig. 4 Instantaneous flame boundary and vorticity contours approaching blowout for the nominal configuration.

boundary of the flame. The strain rate along the boundary of the flame is determined from:

$$
\kappa=-n_{x} n_{y}\left(\frac{\partial v}{\partial x}+\frac{\partial u}{\partial y}\right)+\left(1-n_{x}^{2}\right) \frac{\partial u}{\partial x}+\left(1-n_{y}^{2}\right) \frac{\partial v}{\partial y}
$$

where $n_{x}$ and $n_{y}$ are the horizontal and vertical normal components along the flame boundary, respectively. It is noted that the strain rate increases as the flame approaches blowout. Large strain rate magnitudes are expected to initiate localized extinction events along the boundary of the flame. Localized extinction can result in flame holes that allow cold reactants to penetrate the recirculation region, reducing the temperature within the bluff body wake. Reduced temperatures within the bluff body wake weaken the overall stabilization mechanism as recirculating products can no longer reignite the reacting shear layers [14]. Since the the regions of high strain also correspond to localized regions of large vorticity magnitudes, it is important to understand the effect of the altering pressure gradients on the flow field vorticity and the strain rate evolution along the flame.

The effects of the pressure gradients upon the flow field vorticity can be analyzed by evaluating the combustion induced vortex mechanisms of dilatation and baroclinic torque. The dilatation is a result of the gaseous expansion of the hot combustion products while the baroclinic torque emerges from the cross product of the downstream pressure gradient and the density gradient normal to the flame brush. The dilatation and baroclinic torque are calculated using equations 2 and 3 respectively.

$$
\begin{gathered}
\vec{\omega} \cdot \nabla \vec{V} \\
\frac{1}{\rho^{2}}(\nabla \vec{\rho} \times \nabla \vec{P})
\end{gathered}
$$

Here, the density gradient $(\nabla \vec{\rho})$, is known from the $\mathrm{C}_{2}^{*}-\mathrm{CH}^{*}$ equivalence ratio data and the pressure gradient $(\nabla \vec{P})$ is calculated for each axial downstream location using the slope of the pressure data $(d P / d x)$ presented in Fig. 3 .

The baroclinic torque is calculated along the boundary of the lower flame edge and propagated through time, providing a spatiotemporal evolution throughout the duration of flame extinction. Dilatation is calculated in a similar manner, however, the outer boundary of the flame is not an adequate location to evaluate the effects of gaseous expansion as the cold reactants have not yet burned within the thickness of the flame. To accommodate, dilatation is quantified within the boundary of the flame at a distance of twice the laminar flame thickness $\left(2 L_{F}\right)$ toward the flow centerline; this is depicted within Fig. 53. The inward shift of the evaluation coordinates captures the regions where entrained reactants will have already burned within the flame thickness. Burned products will have a higher temperature and a lower density than the cold reactants and therefore accelerate within the flow field. In this manner, the effects of dilatation can be better captured within the domain of the accelerating products.

The spatial and temporally averaged vorticity mechanisms are depicted within Fig. 5b. The vorticity mechanisms are evaluated using the lower portion of the flame boundary and the spatiotemporal mean is calculated utilizing the data points between between $3 \leq x / H \leq 4$ and averaged through the duration of the extinction process to obtain a single value for each pressure configuration. This gives an overall idea of how the altering pressure gradients effects the combustion induced vorticity mechanisms within the extinguishing flow field. It is evident that as the global pressure gradient becomes stronger, the combustion induced vorticity mechanisms also increase in magnitude. Also note that the 
magnitude of baroclinic torque vorticity is much larger than the magnitude of the dilatation near the boundary of the flame. For this reason, the baroclinic torque will have a larger impact on the flow field vorticity dynamics.

To fully characterize the evolution of the baroclinic torque throughout the extinction process, Fig. 5; shows the normalized baroclinicity resolved along the lower boundary of the flame through time and space. The baroclinic torque magnitudes are shown to decrease through time across all axial locations within the immediate bluff body wake. The decreased baroclinic torque magnitudes will affect the vorticity within the flow field as well as the strain rates experienced by the flame boundary.

To understand the effects of the baroclinic torque magnitudes on the flame stability, Fig. 6 shows the temporal evolution of the vorticity and the strain rate along the flame at $x / H=3$. For all configurations, the temporal reduction of baroclinic torque produced by the flame correlates with an increase of both the strain rate and vorticity magnitudes experienced by the flame. Additionally, both quantities are shown to experience an oscillatory behavior with a time-averaged increase when approaching blowout. It is also noticed that the vorticity and strain rate magnitudes experienced by the nozzle configuration are smallest throughout much of the time duration of extinction. Conversely, the diffuser configuration tends to experience the largest vorticity and strain rate magnitudes through time. This indicates that the larger magnitudes of baroclinic torque generated by the nozzle configuration help reduce the strain rate magnitudes experienced by the flame and hinder the extinction process.

(A)

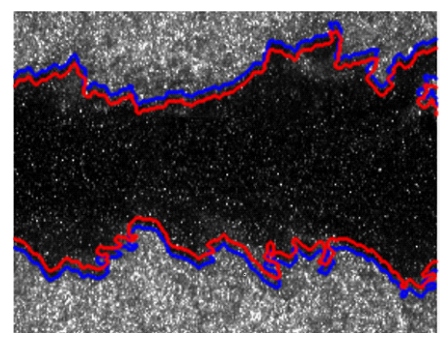

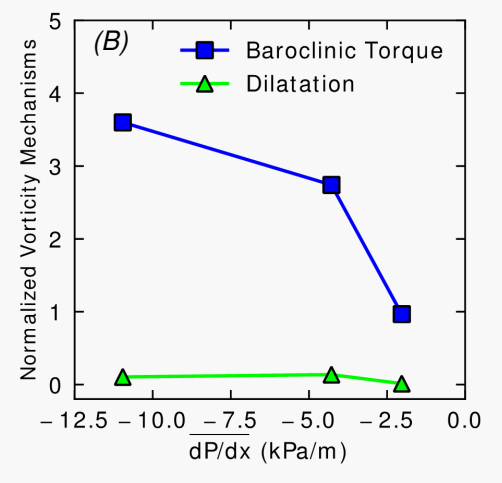

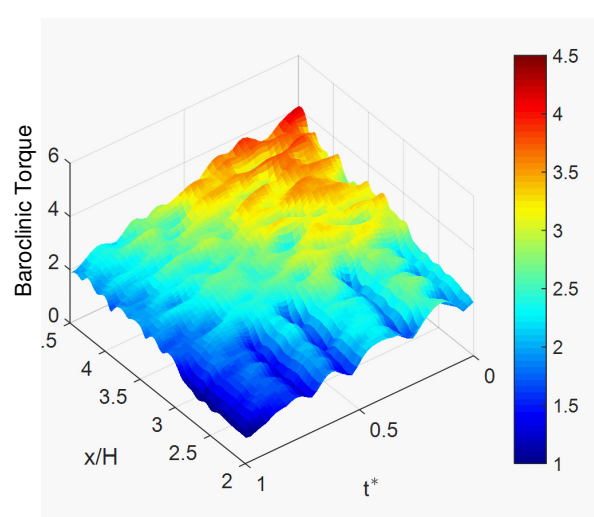

Fig. 5 Combustion induced vorticity mechanisms throughout flame extinction. (A) PIV image with flame trace (blue) and shifted flame coordinates (red) where dilatation is calculated. (B) Spatially and temporally averaged vorticity mechanisms for three wall configurations. Vorticity mechanisms are normalized by $\left(H L_{F} / U_{l i p} S_{L}\right)$. (C) Spatially and temporally resolved baroclinic torque throughout extinction for the nominal configuration.

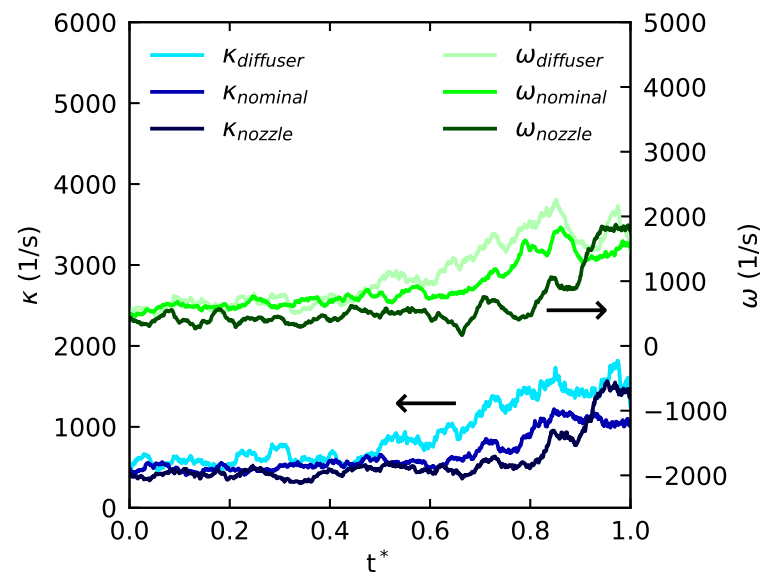

Fig. 6 Temporal strain rate and vorticity along the lower flame boundary at $x / H=3$. 


\section{Conclusion}

The current study presents the effects of changing the downstream pressure gradient on lean flame extinction. The downstream pressure gradient is manipulated by altering the upper and lower wall configurations of the combustion test section. Results have indicated that as the axial pressure gradient is altered, the magnitudes of the combustion induced vortex mechanisms of dilatation and baroclinic torque change accordingly. However, the magnitudes of baroclinic torque are significantly larger than the dilatation for all pressure gradient configurations. To expand upon this, the spatial and temporal baroclinicity produced by the flame is presented throughout the extinction process to gain an understanding of the evolution of the vorticity within the extinguishing flow field. Finally, the local strain rate and vorticity characteristics are investigated to understand how they are influenced by the temporally decreasing baroclinic torque. It was found that the stronger pressure gradient attenuated the vorticity magnitudes experienced by the flame and also educed the strain rate magnitudes experienced by the flame. In this manner, the stronger pressure gradient induced by the nozzle provides better flame stability than the nominal or diffuser configurations.

\section{Acknowledgments}

The work is sponsored by the Air Force Office of Scientific Research (FA9550-16-1-0403, Program Manager: Dr. Chiping Li). This material is based upon work supported by the Air Force Office of Scientific Research under award number FA9550-16-1-0403 and FA9550-16-1-0044

\section{References}

[1] Lovett, J., Brogan, T., Philippona, D., Kiel, B., and Thompson, T., "Development needs for advanced afterburner designs," 40th AIAA/ASME/SAE/ASEE Joint Propulsion Conference and Exhibit, 2004, p. 4192.

[2] Lovett, J. A., Ahmed, K., Bibik, O., Smith, A. G., Lubarsky, E., Menon, S., and Zinn, B. T., "On the influence of fuel distribution on the flame structure of bluff-body stabilized flames," Journal of Engineering for Gas Turbines and Power, Vol. 136, No. 4, 2014, p. 041503.

[3] Candel, S., "Combustion dynamics and control: Progress and challenges," Proceedings of the combustion institute, Vol. 29, No. 1, 2002, pp. 1-28.

[4] Ozawa, R., "Survey of basic data on flame stabilization and propagation for high speed combustion systems," The Marquart Co., TR AFAPL-TR-70-81, 1971.

[5] Herbert, M., "Aerodynamic influences on flame stability," Progress in Combustion Science and Technology, Elsevier, 1960, pp. 61-109.

[6] Ebrahimi, H., "Overview of gas turbine augmentor design, operation, and combustion oscillation," 42nd AIAA/ASME/SAE/ASEE Joint Propulsion Conference \& Exhibit, 2006, p. 4916.

[7] Roshko, A., "On the wake and drag of bluff bodies," Journal of the aeronautical sciences, Vol. 22, No. 2, 1955, pp. $124-132$.

[8] Longwell, J. P., Frost, E. E., and Weiss, M. A., "Flame stability in bluff body recirculation zones," Industrial \& Engineering Chemistry, Vol. 45, No. 8, 1953, pp. 1629-1633.

[9] Zukoski, E. E., and Marble, F. E., "Experiments concerning the mechanism of flame blowoff from bluff bodies," 1983.

[10] Kiel, B., Garwick, K., Lynch, A., Gord, J., and Meyer, T., "Non-reacting and combusting flow investigation of bluff bodies in cross flow," 42nd AIAA/ASME/SAE/ASEE Joint Propulsion Conference \& Exhibit, 2006, p. 5234.

[11] Geikie, M. K., Carr, Z. R., Ahmed, K. A., and Forliti, D. J., "On the Flame-generated Vorticity Dynamics of Bluff-body-stabilized Premixed Flames," Flow, Turbulence and Combustion, Vol. 99, No. 2, 2017, pp. 487-509.

[12] Hamlington, P. E., Poludnenko, A. Y., and Oran, E. S., "Interactions between turbulence and flames in premixed reacting flows," Physics of Fluids, Vol. 23, No. 12, 2011, p. 125111.

[13] Shanbhogue, S. J., Husain, S., and Lieuwen, T., "Lean blowoff of bluff body stabilized flames: Scaling and dynamics," Progress in Energy and Combustion Science, Vol. 35, No. 1, 2009, pp. 98-120.

[14] Lieuwen, T., Shanbhogue, S., Khosla, S., and Smith, C., "Dynamics of bluff body flames near blowoff," 45th AIAA Aerospace Sciences Meeting and Exhibit, 2007, p. 169. 
[15] Emerson, B., O'Connor, J., Juniper, M., and Lieuwen, T., "Density ratio effects on reacting bluff-body flow field characteristics," Journal of Fluid Mechanics, Vol. 706, 2012, pp. 219-250.

[16] Chaudhuri, S., Kostka, S., Renfro, M. W., and Cetegen, B. M., "Blowoff dynamics of bluff body stabilized turbulent premixed flames," Combustion and flame, Vol. 157, No. 4, 2010, pp. 790-802.

[17] Tuttle, S. G., Chaudhuri, S., Kopp-Vaughan, K. M., Jensen, T. R., Cetegen, B. M., Renfro, M. W., and Cohen, J. M., "Lean blowoff behavior of asymmetrically-fueled bluff body-stabilized flames," Combustion and flame, Vol. 160, No. 9, 2013, pp. $1677-1692$.

[18] Geikie, M. K., and Ahmed, K. A., "Lagrangian mechanisms of flame extinction for lean turbulent premixed flames," Fuel, Vol. 194, 2017, pp. 239-256.

[19] Nair, S., and Lieuwen, T. C., "Near-blowoff dynamics of a bluff-body stabilized flame," Journal of Propulsion and power, Vol. 23, No. 2, 2007, pp. 421-427.

[20] Sankaran, V., Palies, P., Liljenberg, S., Teerlinck, K., and Soteriou, M., "Stabilization dynamics of bluff-body premixed flames," 50th AIAA Aerospace Sciences Meeting including the New Horizons Forum and Aerospace Exposition, 2012, p. 352.

[21] Erickson, R., and Soteriou, M., "The influence of reactant temperature on the dynamics of bluff body stabilized premixed flames," Combustion and Flame, Vol. 158, No. 12, 2011, pp. 2441-2457.

[22] Mehta, P., and Soteriou, M., "Combustion heat release effects on the dynamics of bluff body stabilized premixed reacting flows," 41 st AIAA Aerospace Sciences Meeting \& Exhibit, Reno, NV, 2003.

[23] Geikie, M. K., Engelmann, C., Morales, A. J., Schale, R., and Ahmed, K., "Turbulence-Flame Interaction in the Thin Reaction Regime," 53rd AIAA/SAE/ASEE Joint Propulsion Conference, 2017, p. 4681.

[24] Geikie, M. K., Morales, A. J., Engelmann, C., and Ahmed, K., “Turbulence-Flame Interaction in the Broken Reaction Regime," 2018 AIAA Aerospace Sciences Meeting, 2018, p. 0162.

[25] Morales, A. J., Geikie, M. K., Engelmann, C., and Ahmed, K., "Development and Characterization of a Turbulence Generator for Low-Speed Wind Tunnel Applications," 53rd AIAA/SAE/ASEE Joint Propulsion Conference, 2017, p. 4966.

[26] Morales, A. J., Geikie, M. K., Engelmann, C., Ahmed, K., and Lasky, I. M., “Turbulence Regime Characterization for Premixed Combustion," 2018 AIAA Aerospace Sciences Meeting, 2018, p. 0160.

[27] Jeong, Y. K., Jeon, C. H., and Chang, Y. J., "Evaluation of the equivalence ratio of the reacting mixture using intensity ratio of chemiluminescence in laminar partially premixed CH4-air flames," Experimental Thermal and Fluid Science, Vol. 30, No. 7, 2006, pp. 663-673.

[28] JOHN, R. R., MARTIN SUMMERFIELD, "Effect of turbulence on radiation intensity from propane-air flames," Journal of Jet Propulsion, Vol. 27, No. 2, 1957, pp. 169-175.

[29] Clark, T., "Studies of $\mathrm{OH}, \mathrm{CO}, \mathrm{CH}$ and $\mathrm{C} 2$ radiation from laminar and turbulent propane-air and ethylene-air flames-NACA Technical Note 4266," Tech. rep., Technical report, National Advisory Committee for Aeronautics, Lewis Flight Propulsion Laboratory Cleveland, Ohio, 1958. 